\title{
The tumor border configuration of colorectal cancer as a histomorphological prognostic indicator
}

\section{Viktor H. Koelzer * and Alessandro Lugli}

Clinical Pathology Division and Translational Research Unit, Institute of Pathology, University of Bern, Bern, Switzerland

\section{Edited by:}

Kenichi Hirabayashi, Tokai University

School of Medicine, Japan

\section{Reviewed by:}

Tomio Arai, Tokyo Metropolitan Geriatric Hospital, Japan; Tokyo Metropolitan Institute of Gerontology, Japan

Takuma Tajiri, Showa University

Fujigaoka Hospital, Japan

\section{*Correspondence:}

Viktor H. Koelzer, Clinical Pathology Division and Translational Research Unit, Institute of Pathology, University of Bern, Murtenstrasse 31, Bern

CH-3010, Switzerland

e-mail: viktor.koelzer@pathology. unibe.ch
Histomorphological features of colorectal cancers (CRC) represent valuable prognostic indicators for clinical decision making. The invasive margin is a central feature for prognostication shaped by the complex processes governing tumor-host interaction. Assessment of the tumor border can be performed on standard paraffin sections and shows promise for integration into the diagnostic routine of gastrointestinal pathology. In aggressive CRC, an extensive dissection of host tissue is seen with loss of a clear tumor-host interface. This pattern, termed "infiltrative tumor border configuration" has been consistently associated with poor survival outcome and early disease recurrence of CRC-patients. In addition, infiltrative tumor growth is frequently associated with presence of adverse clinicopathological features and molecular alterations related to aggressive tumor behavior including BRAFV600 mutation. In contrast, a well-demarcated "pushing" tumor border is seen frequently in CRC-cases with low risk for nodal and distant metastasis. A pushing border is a feature frequently associated with mismatch-repair deficiency and can be used to identify patients for molecular testing. Consequently, assessment of the tumor border configuration as an additional prognostic factor is recommended by the AJCC/UICC to aid the TNM-classification. To promote the assessment of the tumor border configuration in standard practice, consensus criteria on the defining features and method of assessment need to be developed further and tested for inter-observer reproducibility. The development of a standardized quantitative scoring system may lay the basis for verification of the prognostic associations of the tumor growth pattern in multivariate analyses and clinical trials. This article provides a comprehensive review of the diagnostic features, clinicopathological associations, and molecular alterations associated with the tumor border configuration in early stage and advanced CRC.

Keywords: tumor border configuration, invasive margin, tumor growth pattern, infiltrative border, pushing border, colorectal cancer, prognostic factor, tumor-host interaction

\section{INTRODUCTION}

Prognostication of colorectal cancer (CRC) is based on histopathological staging of the resection specimen according to the AJCC/UICC TNM-classification and guides treatment decisions. The TNM-classification requires reporting of local tumor extent, status of regional nodes, lymphatic and blood vessel invasion, and residual tumor as essential prognostic factors (1). Additional histomorphological indicators are tumor grade, tumor budding, and tumor border configuration (1). Even though the TNMclassification is the gold standard in clinical practice, some patients with lower TNM-stages show a worse prognosis compared to patients with a higher tumor stage. This holds true for selected stage I CRC-patients, some of which paradoxically relapse with nodal metastasis after removal of an early invasive lesion (2). Identification of these patients for segmental resection is of paramount importance for a risk-adapted treatment approach. Further, locally advanced nodal-negative stage II CRC can behave aggressively in the presence of additional histomorphological risk factors $(3,4)$. In particular, nodal-negative stage II patients with serosal perforation, lymphovascular invasion, perineural infiltration, or an invasive tumor border configuration may have a comparable outcome to stage III patients with nodal positive disease, but do not yet receive neoadjuvant treatment as a standard of care (5-7).

Several recent clinical studies have explored the use of chemotherapeutic treatment in stage II patients with mixed results. A meta-analysis of the available literature by the American Society of Clinical Oncology (ASCO) failed to identify a significant benefit in overall survival of stage II patients receiving adjuvant therapy (8). Consequently, the ASCO does not recommend routine use of chemotherapy in stage II CRC-patients. However, use of adjuvant therapy is considered justified in well-informed patients with additional risk factors such as inadequately sampled nodes, T4 lesions, perforation, or poorly differentiated histology (8). The recent QUASAR trial provides further evidence of an incremental benefit of adjuvant therapy in stage II patients with a significant decrease in disease recurrence and a 3-6\% improvement in survival in patients receiving adjuvant chemotherapy (4). From the pathologist's perspective, the recognition, standardization, and reporting of histomorphological prognostic features are an important basis for identification of patient subgroups with a higher risk 

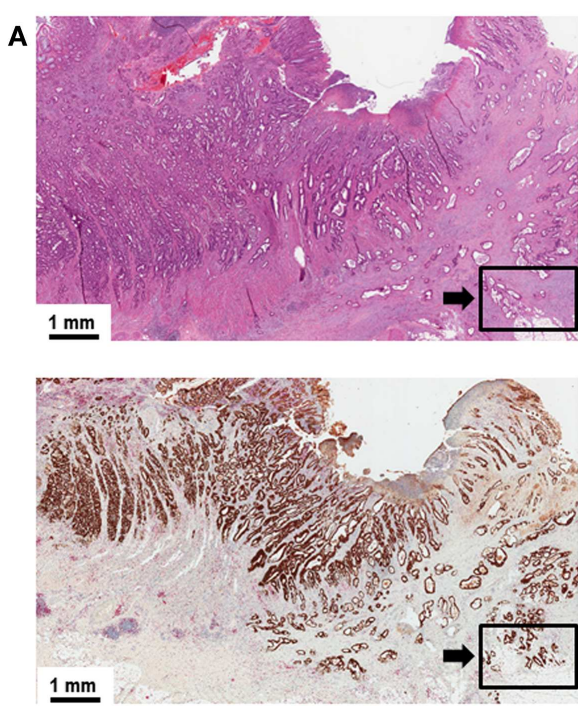

FIGURE 1 | (A) Low power image $(5 x)$ of a H\&E slide and pan-cytokeratin (brown)/CD8 (red) double stain illustrating a transmurally invasive primary $\mathrm{CRC}$ with an infiltrative tumor border configuration. Delineation of tumor and host tissue is difficult in the H\&E stain. Residual host tissue is present between infiltrating cords and sharp wedges of long-stretched tumor glands.
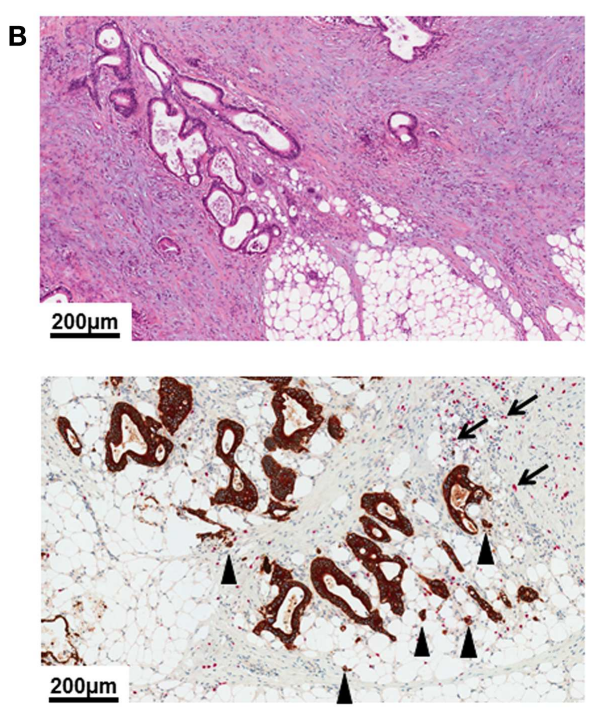

(B) High power detail $(20 \times)$ of (A). Note the diffuse dissection of irregularly shaped tumor glands through the mesenteric adipose tissue. CD8+ lymphocytes at the tumor invasive front are infrequent (arrows). Tumor buds invading the stroma ahead of the invasive front can be identified as a superimposed feature (arrow heads). of recurrence (9). Clinically, these patients may then benefit from individualized therapeutic approaches and enrollment in clinical trials.

This article provides a comprehensive review on the pathology, biology, and prognostic value of tumor border configuration in CRC. An irregular, "infiltrative" advancing edge is a hallmark of highly aggressive tumors and has been classified as an independent adverse prognostic indicator and may predict propensity for systemic spread of disease in colon and rectal cancer (1016). In contrast, tumors demonstrating a smooth demarcation with a rounded infiltrative border are classified as having an "expansile" or "pushing" border configuration. As will be discussed in detail, this feature is predictive of limited tumor aggressiveness and is often seen in mismatch-repair (MMR) deficient CRCs (17-19). The addition of tumor border configuration to TNM-staging may help to stratify CRC-patients of a given stage into diagnostic subgroups (7). Importantly, this adverse prognostic feature can be easily detected on standard H\&E slides by the histopathologist and is recommended for routine reporting of transmurally invasive CRC as a category IIB prognostic factor (20).

\section{SURGICAL PATHOLOGY}

The histomorphological variance of the tumor border configuration of CRC was first described by Jass in 1986 as an important histomorphological prognostic indicator in rectal cancer patients (21). Methodologically, tumor border configuration is a feature diagnosed at low magnification and must be clearly differentiated from diagnostic features seen at high power such as tumor budding (10). According to Jass, an infiltrative border configuration should already be suspected when examination of the histopathologic slide with the naked eye does not allow a clear definition of the invasive margin and it seems impossible to resolve host tissue from malignant glands (Figure 1A) (21-23). At low magnification, tumors with an infiltrative growth pattern show dissection of tumor tissue through the anatomic structures of the bowel wall with little or absent desmoplastic stromal response (21-23). The dissecting tumor glands often form irregular clusters or cords of cells, long-stretched glandular structures, or sharp wedges leaving residual host tissue in between, a pattern termed "streaming dissection" (Figure 1B) $(10,11,23,24)$. Presence of perineural invasion on the histologic slide is a further indicator of diffuse infiltration (23). In contrast, a pushing tumor border configuration should be suspected when naked eye examination of the histologic slide allows a clear delineation of the tumor invasive front and host tissue (Figure 2A). Under low magnification, a round "circumscribed" configuration of the infiltrative margin is characteristic of the "pushing" pattern of infiltration (23). Widely dissecting tumor glands in the muscularis propria or mesenteric adipose tissue are absent (Figure 2B).

Infiltrative growth is often observed as a heterogeneously distributed feature in CRC and a majority of cases demonstrate a predominant or at least focally infiltrative tumor border configuration, leading to variability in interpretation. According to Jass, the tumor border configuration is classified as either infiltrating or pushing in a two-tier system (22). This recommendation has been adapted in the CAP reporting standards for CRC (11). However, other authors have advocated the use of a trichotomous classification and to restrict the "infiltrating" pattern to cases with an unequivocal infiltrating growth involving the complete tumor border (25). All of the criteria used to differentiate the pushing from the infiltrative tumor border configuration share a subjective, 

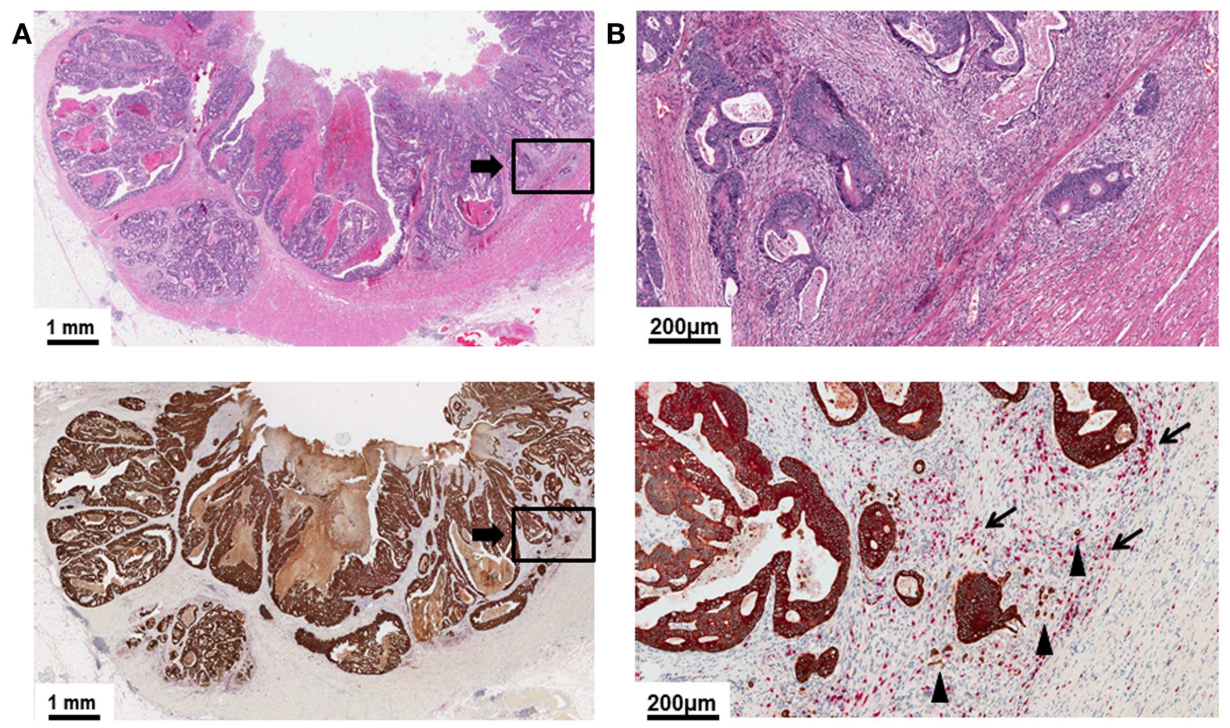

FIGURE 2 | (A) Low power image $(5 \times)$ of a H\&E slide and pan-cytokeratin (brown)/CD8 (red) double stain demonstrating a primary CRC with a pushing tumor border configuration of growth. The tumor border is round and well-recognizable at low magnification. Tumor and host tissue can be easily differentiated. Host tissue is displaced by expansile tumor growth. (B) High power detail $(20 \times)$ of (A). CD8+ lymphocytes are commonly observed (arrows). Tumor budding can be recognized as a superimposed feature but is generally infrequent (arrow heads). qualitative nature, and a hierarchy of features have not been designated. This may lead to a very variable classification of cases showing a mixed morphology with either the infiltrating or pushing subset. Further, there is currently no clear consensus on the minimum extension of the infiltrative component necessary to designate a case as having an infiltrative tumor border configuration. While some authors require a predominant infiltrative growth pattern for this classification and describe as few as $17 \%$ of CRCs as infiltrative, others have included any case with at least focal infiltrative growth pattern assigning up to $70 \%$ of CRC-cases to the infiltrative group $(7,13)$. Consequently, a suboptimal interobserver reproducibility has been described for the assessment of tumor border configuration according to the Jass criteria, but can be improved by educating the observers to the defining features (23). Thus, a standardization of the pathologic quantification of the invasive growth pattern is urgently needed to advance its use in daily diagnostic practice.

Tumor budding, defined as the presence of single cells or small clusters of up to five cells ahead of the invasive front, is frequently observed as a superimposed pattern in cases with an infiltrative tumor border configuration (Figure 3) (26). However, tumor budding is a separate, independent feature observed at high magnification and must not be used to differentiate infiltrative from pushing tumor growth (9). The presence of tumor budding is an increasingly important histomorphological prognostic factor in CRC $(11,24,27)$. Biologically, tumor budding is likely the visible correlate of the process of epithelial mesenchymal transition, during which cancer cells, epithelial by nature, acquire mesenchymal characteristics with capability for migration, stromal lysis, and vascular invasion (28). In early stage CRC, the presence of tumor budding can be indicative of clinical undetectable micrometastases

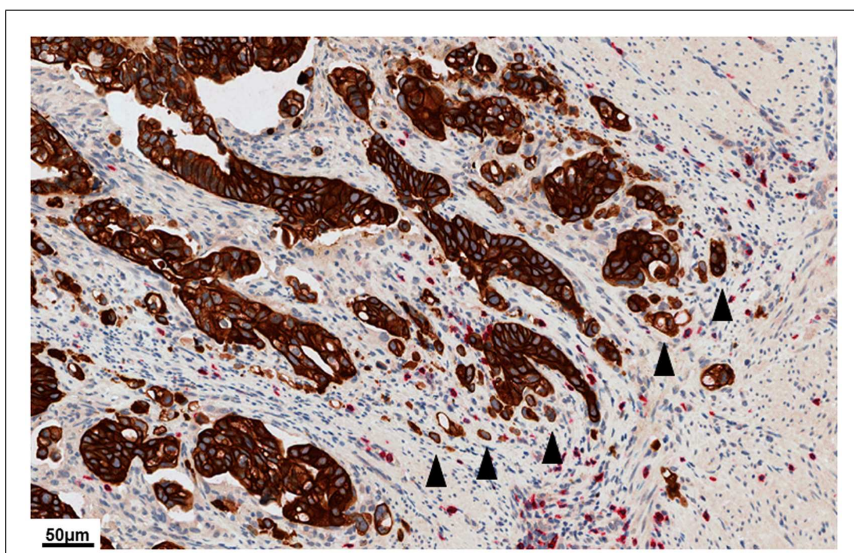

FIGURE 3 | High power image (40x) of a pan-cytokeratin (brown)/CD8 (red) double stain illustrating dense tumor budding at the tumor invasive front. Tumor buds (arrow heads) are defined as single cells or small clusters of up to five cells ahead of the tumor invasive front. High-grade tumor budding is a feature of aggressive biological behavior in colorectal cancer. Even though tumor budding is more frequently observed in cases with an infiltrative tumor border configuration, this is an independent feature observed at high power and must not be used to define the quality of the tumor border.

present already at the time of resection of the primary tumor (29). In locally advanced CRC, the presence of tumor budding is an important prognostic indicator for a reduced 5-year survival outcome with elevated risk for disease relapse (27, 30-34). Consequently, tumor budding has been designated a category IIB prognostic factor by the CAP (20). 


\section{PROGNOSTIC IMPACT \\ EARLY STAGE CRC}

Early stage CRC is treated by endoscopic or surgical resection as standard of care. Consequently, the prediction of local recurrence and nodal metastasis by assessment of histopathological features of early invasive CRC is of central importance for patient treatment and follow-up. Several retrospective correlative studies have addressed the prognostic value of the tumor border configuration in early stage CRC (Table 1) and have reported conflicting evidence: in curatively resected submucosally invasive CRC, both Egashira $(n=140)$ and Wang $(n=159)$ failed to identify an association of tumor growth pattern with nodal metastasis $(32,35)$. In contrast, Keum and colleagues characterize an infiltrating pattern of growth as opposed to a smooth pushing border as a significant independent predictor for disease recurrence in an analysis of 434 patients who underwent curative resection for stage I CRC (36). Further, in an analysis of 111 submucosally invasive pT1 CRCcases, Akishima-Fukasawa et al. describe the invasive tumor border configuration to be a highly significant predictor of regional lymph node metastasis in univariate but not in multivariate analysis (37). This data is expanded for stage I patients with muscularis propria invasion by Kajiwara and associates who demonstrate an increased frequency of nodal involvement in patients with an infiltrative tumor border configuration in univariate but not in multivariate analysis (38). Taken together, there is a lack of conclusive data on the prognostic value of invasive border configuration in early stage CRC. This may be due to absence of standardized criteria for pathologic assessment. Further, the assessment of the predominant growth pattern in early invasive CRC may be restricted because of limited invasive tumor tissue. As a result, the recommendation for the assessment of tumor border configuration by the AJCC is currently limited to transmurally invasive CRCs (20).

\section{ADVANCED CRC}

In transmurally invasive CRC, an infiltrative tumor border configuration has been established as an adverse prognostic factor in several well-designed retrospective cohort studies including CRCpatients of all stages (Table 2). In detail, assessment of the tumor border configuration was found to provide stage-independent prognostic information on the relative risk for disease recurrence and cancer-related death after resection of the primary tumor aiding the TNM- and Dukes-classification (7, 12, 13, 15, 25, 26, $39,40)$. The prognostic value of tumor border configuration in locally advanced CRC is supported by several multivariate analyses on independent patient cohorts leading to a recommendation for routine reporting by the CAP (20). Recent data also indicate that the prognostic value of the tumor growth pattern in stage I-III CRC is independent of central molecular features including KRAS, BRAF, and PIK3CA mutations as well as MSI-status and CIMP methylation (25). Assessment of the tumor border configuration should therefore be included in daily diagnostic practice to aid selection of high risk CRC-patients that could derive the most benefit from adjuvant multi-agent therapy.

Several studies have also analyzed the prognostic value of tumor border configuration in locally advanced CRC stratified by stage. The results suggest that additional prognostic information is carried by this feature in patients with stage II, nodal-negative CRC.
In a cohort of 238 well-characterized stage IIA CRC-patients, Cianchi et al. provide comprehensive evidence of a significantly elevated risk of cancer-specific death in patients with an infiltrative vs. pushing tumor border configuration: 5-year survival rates are specified as $81.8 \%$ with an infiltrating tumor border as opposed to $92.9 \%$ in cases demonstrating an expansile growth pattern $(p<0.01)$ (43). Ueno and colleagues confirm these findings in a study of 994 CRC-cases including 434 stage II and 560 stage III patients reporting a 5-year survival rate of $81.8 \%$ for the infiltrating group, compared to $92.9 \%$ when an expansile border configuration was recorded $(p=0.015)$ (39). This preliminary evidence suggests that the assessment of tumor border configuration may be a promising additional risk-indicator in stage II disease. Assessment of this feature could aid the selection of high risk stage II patients for adjuvant multi-agent therapy and should be investigated further in large randomized multi-center trials to build a reliable evidence base.

The prognostic value of the tumor border configuration in advanced CRC is further supported by correlation with vascular invasion, which is another established feature of aggressive disease. In an analysis of 994 stage II-III patients, Ueno and colleagues describe a strong relation of an infiltrative growth pattern in the muscularis propria to the presence of hemangioinvasion at the invasive front of CRC (39). This data is expanded in a study of 427 stage I-IV CRC-patients by Zlobec and associates who describe a significantly increased frequency of vascular invasion in tumors with an infiltrative growth pattern (40). Further, tumor border configuration was identified as a decisive classifier for the identification of tumors with vascular invasion when combined with expression of Raf-kinase inhibitor protein (RKIP), urokinase plasminogen activator receptor ( $\mu \mathrm{PAR})$, and the proliferative index. As RKIP and $\mu$ PAR are important biomarkers of tumor motility and invasive capacity, this provides further evidence for an elevated risk of distant metastasis predicted by the tumor growth pattern. Interestingly, an infiltrative tumor border configuration is also strongly predictive of high-grade tumor budding, indicating that the biological processes leading to the formation of tumor buds and an invasive tumor border configuration may be inter-related $(39,44)$. Patients presenting with both high-grade tumor budding and an infiltrative tumor border configuration have an increased risk of cancer-related death than patients presenting with either alone (39).

Taken together, a solid evidence base for the prognostic value of tumor border configuration exists in advanced CRC and standard reporting of this feature is recommended for diagnostic practice (20). Nevertheless, in order to definitively evaluate the prognostic importance of tumor border configuration in CRC, statistically robust studies with multivariate analysis and standardized criteria for pathologic assessment will be required, particularly for the stage II subset.

\section{BIOLOGICAL ASPECTS}

The invasive front of CRC embodies the tumor-host interface. Thus, it can be assumed that the histomorphological appearance of this region is the result of the interaction between host and tumor-related factors. Specifically, the dynamics of tumor cell proliferation and expansion is likely governed by specific molecular 


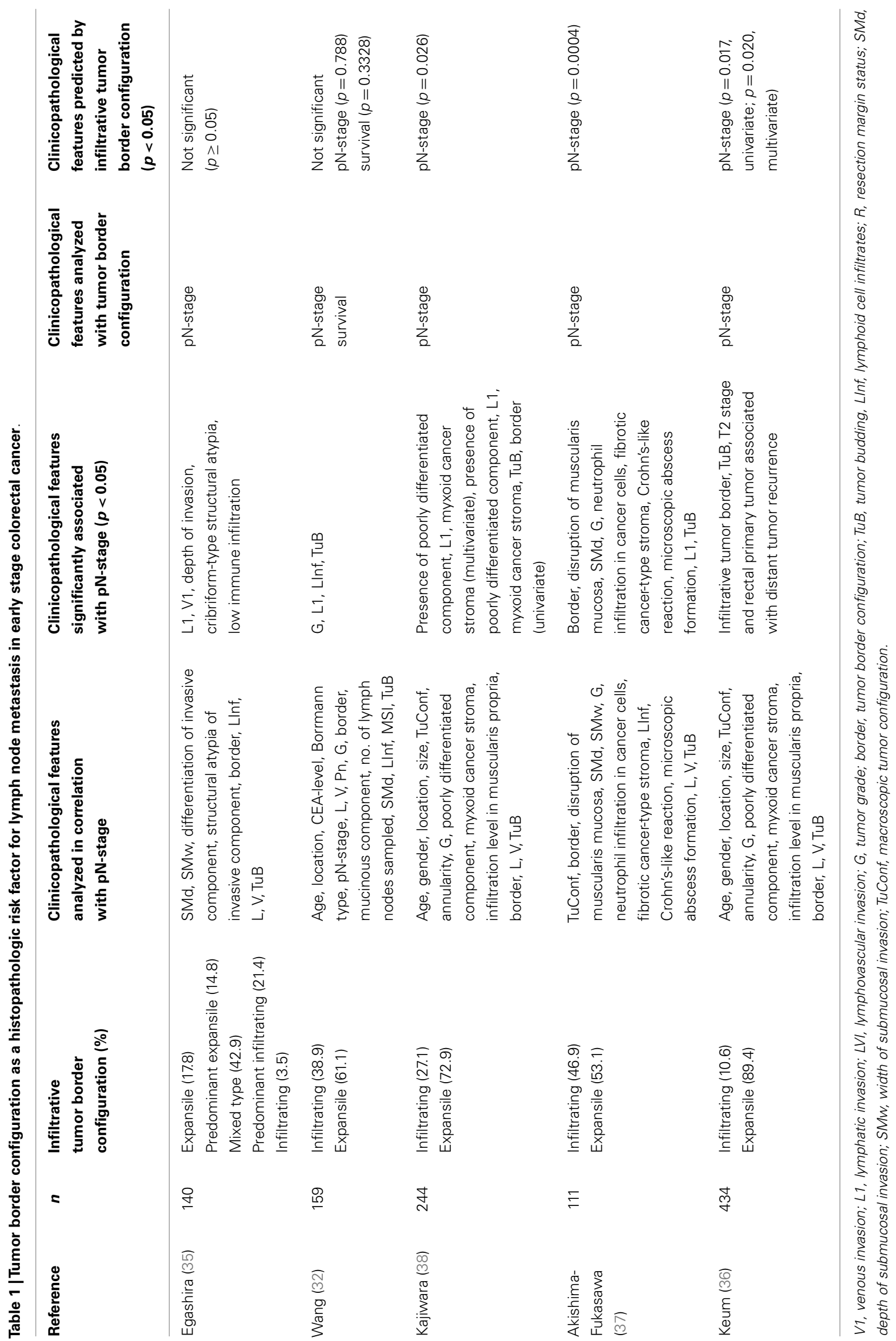




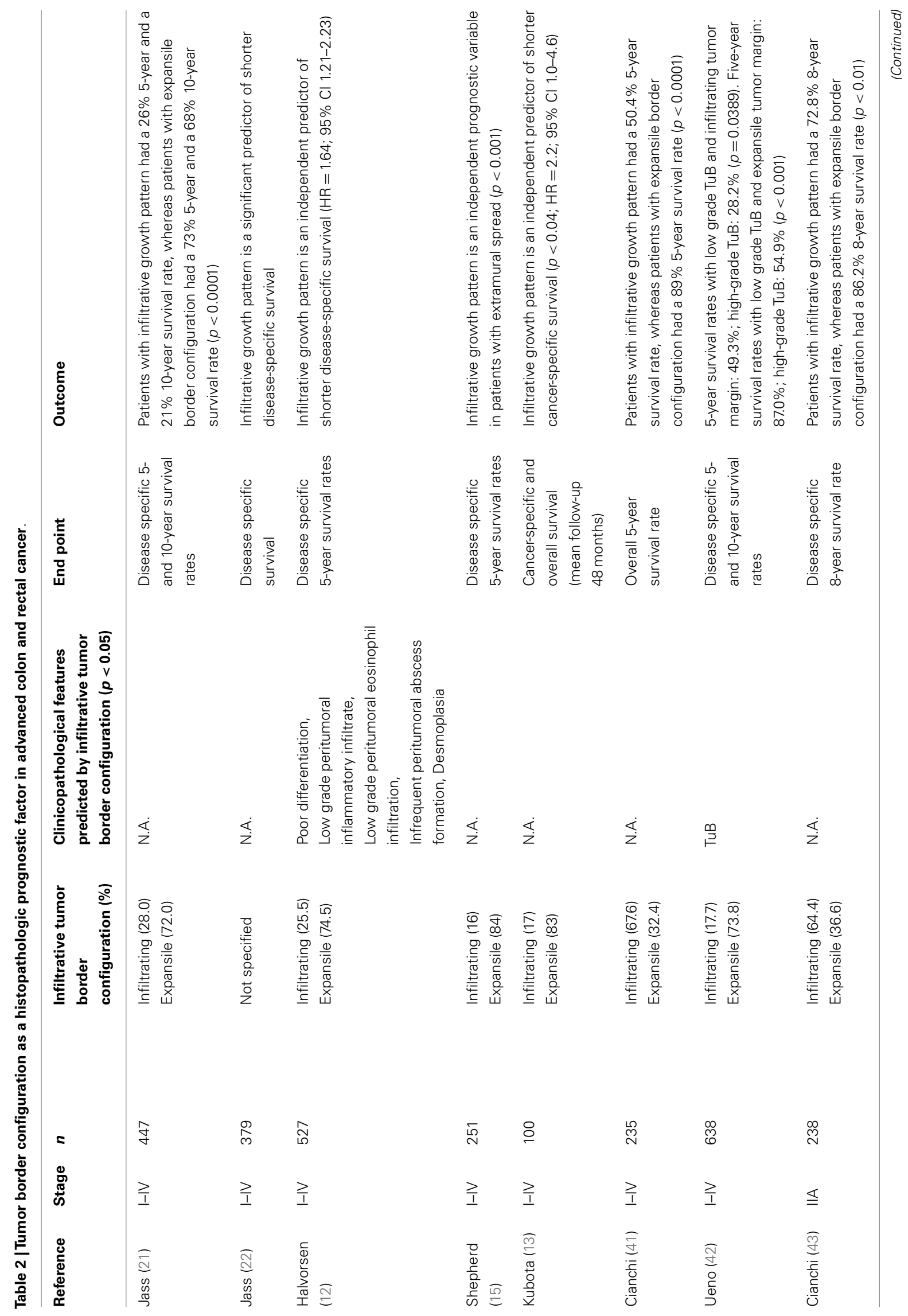




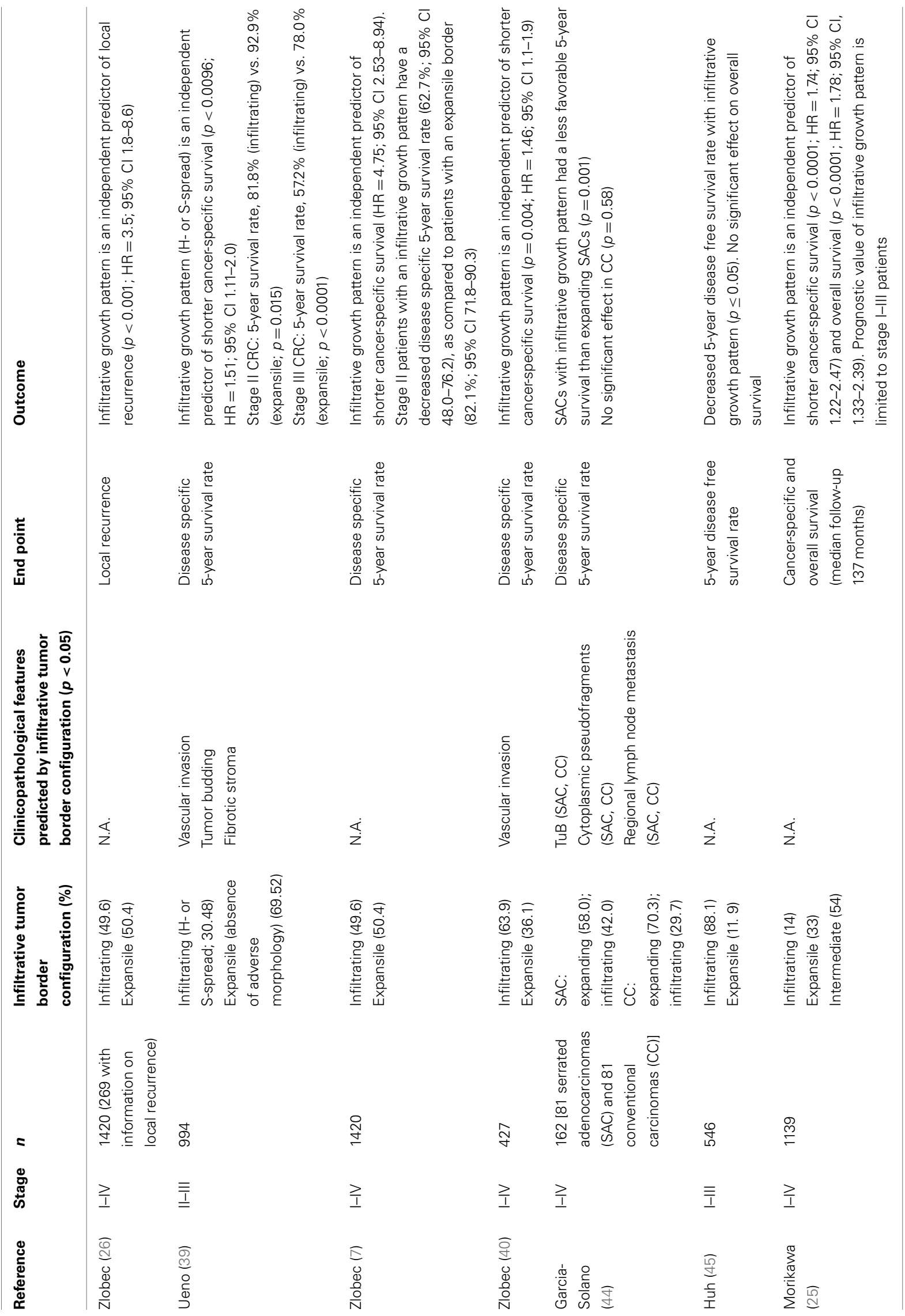


alterations in cancer cells (46): the individual appearance of the tumor invasive margin will reflect the capacity of tumors to proliferate, migrate, and invade the surrounding stroma. An invasive tumor margin may therefore be the morphological snapshot of directed migration of tumor cells into host tissue after division (47). As tumor cell crowding inhibits growth and proliferation, it can be expected that directed growth also correlates with higher rates of tumor expansion (48). In contrast, a pushing margin may represent the random arrangement of tumor cells after division leading to the formation of static round tumor cell clusters with less propensity or capability for invasion of host tissue. Remarkably, primary CRC with a pushing margin also preferentially cause capsulated liver metastasis while cases with infiltrative tumor growth tend to form poorly demarcated lesions indicating that the configuration of the tumor margin could be a conserved tumor feature independent of the surrounding type or quality of host tissue (49). Biologically, the formation of an infiltrative margin may therefore be tantamount to the tendency for "directed tumor growth" while a pushing margin could be the visual correlate of "non-directed growth" in CRC.

Interestingly, the configuration of the invasive margin correlates with some of the well-characterized molecular alterations in CRC. Specifically, a well-demarcated, expansile tumor border is a feature frequently seen in MMR-deficient CRC-cases, particularly in the setting of the hereditary non-polyposis colorectal cancer syndrome (HNPCC) (17-19). As only about $15 \%$ of CRC-cases follow the microsatellite-instable pathway, morphological features are a valuable tool to specifically select cases for molecular testing $(10,18,50)$. If a well-defined pushing margin in addition to mucinous differentiation, solid growth pattern, presence of intraepithelial lymphocytes, and Crohn'slike lymphocytic reaction is observed in a case with rightsided location, the threshold for testing of microsatellite instability should be particularity low as these morphological features reach a sensitivity of $78 \%$ and specificity of up $93 \%$ for MMR-deficiency (18). In contrast, an infiltrating tumor growth pattern is significantly more frequent in tumors with activating BRAF-mutations, while no impact of KRAS-mutations was observed (25). While MMR-deficient CRC generally has a favorable outcome, BRAF is an independent predictor of an aggressive clinical course (51-53). Interestingly, early functional data indicates that constitutive activation of BRAF may increase the migratory and invasive capacity of human colon cancer cells (54). This could contribute to the significantly more frequent invasive growth pattern observed in CRC-cases with activating BRAF-mutations.

Host-related factors may also influence the appearance of the tumor border in CRC. In a well-designed retrospective study of 527 patients, Halvorsen et al. describe a marked absence of peritumoral inflammation in patients with an infiltrative tumor border configuration (12). In contrast, CRC-cases with a pushing border have a well-characterized association with dense peritumoral inflammatory infiltrates (17-19). Interestingly, it is now well-known that the quantity and quality of peritumoral inflammatory infiltrates is a significant independent predictor of survival outcome and related to the efficiency of the anti-tumoral host response, which may be a possible confounding factor of the prognostic benefit of a pushing tumor border configuration (55-57). Additionally, both features are characteristic of MMR-deficiency, which may be a further hidden variable in early reports (17$19)$. Both points have been addressed in recent studies. In an analysis of MMR-proficient 269 patients, Zlobec and colleagues demonstrate that an infiltrative tumor border configuration maintains association with local recurrence of CRC independently of the quantity of CD8+ tumor-infiltrating lymphocytes (TIL) and nodal status (26). In detail, node-negative, TIL-negative patients with an infiltrative border configuration had a probability of 0.55 for recurrence whereas patients with a pushing border had a significantly reduced probability of disease relapse of 0.26 (26). In a study of 1139 well-characterized CRC-patients, Morikawa and associates recently confirmed the absence of a modifying effect of peritumoral lymphocytic infiltration on the prognostic value of the tumor growth pattern (25).

Taken together, the tumor border is shaped both by tumor- and host-related factors. A strong correlation with specific molecular alterations has been described, providing further evidence that specific molecular alterations may dictate the growth dynamics of CRC. Importantly, specific BRAF-mutations and MMR-deficiency are also predictive of response to targeted therapy with 5-FU and Cetuximab-based therapy (58-60). Apart from the well-described prognostic associations the tumor growth pattern could therefore also represent a surrogate morphological indicator for specific molecular mutations with predictive value.

\section{CONCLUSION}

Evaluation of the tumor border configuration by the histopathologist identifies distinct subsets of primary CRC (Table 3) and represents an important histomorphological prognostic indicator. It can be assessed on standard H\&E slides and is consistently related to survival outcome of patients with transmurally invasive $\mathrm{CRC}$ in statistically robust studies and multivariate analysis. However, the configuration of the tumor border is rarely reported in daily diagnostic practice as its significance for prognostication in CRC is not commonly recognized. Further, the subjective nature and lack of standardization of the assessment method may limit frequent reporting of this important feature in clinical practice. The assessment of the tumor border configuration according to the Jass criteria may profit from the development of a standardized quantitative system and needs to be tested further for reproducibility. Methodologically, this may improve the current substantial variation in assessment criteria between different authors and may lay the basis for inclusion of tumor border configuration as a feature for risk assessment in prospective randomized clinical trials.

In early invasive disease, recent studies identify promising associations with clinical outcome. However, evaluation in large, randomized multi-center studies will be needed to conclusively address possible associations of tumor growth pattern with outcome in stage I CRC. For stage II CRC, the evidence base is more consistent making the tumor growth pattern a promising marker to identify high risk patients with locally advanced nodalnegative disease for individualized therapy approaches. However, 
Table 3 | Contrasting features of primary colorectal cancers depending on the tumor border configuration.

\begin{tabular}{|c|c|c|}
\hline Characteristics & Infiltrative & Pushing \\
\hline Examination of the histopathologic & No clear definition of the invasive margin is possible (23) & Clearly recognizable invasive margin \\
\hline slide with the naked eye & Difficulty in resolving host tissue from malignant glands (23) & Easy delineation of host and malignant tissue \\
\hline \multirow[t]{4}{*}{ Low power magnification } & $\begin{array}{l}\text { Dissection of tumor tissue through anatomic structures of } \\
\text { the bowel wall with little or absent desmoplastic stromal } \\
\text { response (23) }\end{array}$ & $\begin{array}{l}\text { "Circumscribed" configuration of the infiltrative } \\
\text { margin with desmoplastic stromal reaction }\end{array}$ \\
\hline & $\begin{array}{l}\text { Irregular clusters, cords, or sharp wedges of long-stretched } \\
\text { tumor glands (23) }\end{array}$ & Absence of widely dissecting tumor glands \\
\hline & Residual host tissue is present between infiltrating glands & Host tissue is displaced \\
\hline & "Streaming dissection" (23) & "Expansile tumor growth" \\
\hline \multirow[t]{3}{*}{ Additional features } & Presence of perineural invasion (23) & Absence of perineural infiltration \\
\hline & High-grade tumor budding is frequently observed (26) & Low grade tumor budding is frequently observed \\
\hline & Peritumoral infiltration less pronounced (12) & Often intense peritumoral inflammation \\
\hline \multirow[t]{2}{*}{ Molecular pathology features } & More frequently observed in cases with activating & More frequently observed in cases with \\
\hline & BRAF-mutations (25) & microsatellite instability (17-19) \\
\hline $\begin{array}{l}\text { Impact on clinical outcome in } \\
\text { transmurally invasive CRC }\end{array}$ & Unfavorable & Favorable \\
\hline
\end{tabular}

verification in statistically robust studies with a specific focus on stage II patients is clearly needed.

The association of tumor growth pattern with molecular features of CRC represents an interesting vantage point for further study. In particular, factors determining the directed growth of CRC cells into host tissue remain to be determined. Further, the influence of BRAF-mutations and MMR-deficiency on tumor growth dynamics and plasticity may provide valuable insights into the biology of CRC.

\section{REFERENCES}

1. Bosman FT, World Health Organization, International Agency for Research on Cancer. Who Classification of Tumours of the Digestive System, 4th Edn. Lyon: International Agency for Research on Cancer (2010).

2. Gockel I, Sgourakis G, Lyros O, Polotzek U, Schimanski CC, Lang H, et al. Risk of lymph node metastasis in submucosal esophageal cancer: a review of surgically resected patients. Expert Rev Gastroenterol Hepatol (2011) 5(3):371-84. doi:10.1586/egh.11.33

3. Schmoll HJ, Van Cutsem E, Stein A, Valentini V, Glimelius B, Haustermans K, et al. Esmo consensus guidelines for management of patients with colon and rectal cancer. A personalized approach to clinical decision making. Ann Oncol (2012) 23(10):2479-516. doi:10.1093/annonc/mds236

4. Quasar Collaborative Group, Gray R, Barnwell J, McConkey C, Hills RK, Williams NS, et al. Adjuvant chemotherapy versus observation in patients with colorectal cancer: a randomised study. Lancet (2007) 370(9604):2020-9. doi:10.1016/S0140-6736(07)61866-2

5. O'Connell JB, Maggard MA, Ko CY. Colon cancer survival rates with the new American joint committee on cancer sixth edition staging. J Natl Cancer Inst (2004) 96(19):1420-5. doi:10.1093/jnci/djh275

6. Santos C, López-Doriga A, Navarro M, Mateo J, Biondo S, Martínez Villacampa M, et al. Clinicopathological risk factors of stage II colon cancer: results of a prospective study. Colorectal Dis (2013) 15(4):414-22. doi:10.1111/ codi. 12028

7. Zlobec I, Baker K, Minoo P, Hayashi S, Terracciano L, Lugli A. Tumor border configuration added to TNM staging better stratifies stage II colorectal cancer patients into prognostic subgroups. Cancer (2009) 115(17):4021-9. doi: $10.1002 /$ cncr. 24450
8. Benson AB III, Schrag D, Somerfield MR, Cohen AM, Figueredo AT, Flynn PJ, et al. American society of clinical oncology recommendations on adjuvant chemotherapy for stage II colon cancer. J Clin Oncol (2004) 22(16):3408-19. doi:10.1200/JCO.2004.05.063

9. Compton CC. Optimal pathologic staging: defining stage II disease. Clin Cancer Res (2007) 13(22 Pt 2):6862s-70s. doi:10.1158/1078-0432.CCR-07-1398

10. Jass JR. Classification of colorectal cancer based on correlation of clinical, morphological and molecular features. Histopathology (2007) 50(1):113-30. doi:10.1111/j.1365-2559.2006.02549.x

11. Compton CC, Fielding LP, Burgart LJ, Conley B, Cooper HS, Hamilton SR, et al. Prognostic factors in colorectal cancer. College of American pathologists consensus statement 1999. Arch Pathol Lab Med (2000) 124(7):979-94. doi:10.1043/0003-9985(2000)124<0979:PFICC>2.0.CO;2

12. Halvorsen TB, Seim E. Association between invasiveness, inflammatory reaction, desmoplasia and survival in colorectal cancer. J Clin Pathol (1989) 42(2):162-6. doi:10.1136/jcp.42.2.162

13. Kubota Y, Petras RE, Easley KA, Bauer TW, Tubbs RR, Fazio VW. Ki-67-determined growth fraction versus standard staging and grading parameters in colorectal carcinoma. A multivariate analysis. Cancer (1992) 70(11):2602-9. doi:10.1002/1097-0142(19921201)70:11<2602::AIDCNCR2820701106>3.0.CO;2-W

14. Quirke P, Dixon MF, Clayden AD, Durdey P, Dyson JE, Williams NS, et al. Prognostic significance of DNA aneuploidy and cell proliferation in rectal adenocarcinomas. J Pathol (1987) 151(4):285-91. doi:10.1002/path.1711510408

15. Shepherd NA, Saraga EP, Love SB, Jass JR. Prognostic factors in colonic cancer. Histopathology (1989) 14(6):613-20. doi:10.1111/j.1365-2559.1989.tb02202.x

16. Fisher ER, Robinsky B, Sass R, Fisher B. Relative prognostic value of the Dukes and the Jass systems in rectal cancer. Findings from the National Surgical Adjuvant Breast and Bowel Projects (Protocol R-01). Dis Colon Rectum (1989) 32(11):944-9. doi:10.1007/BF02552270

17. Klarskov L, Holck S, Bernstein I, Nilbert M. Hereditary colorectal cancer diagnostics: morphological features of familial colorectal cancer type $\mathrm{X}$ versus lynch syndrome. J Clin Pathol (2012) 65(4):352-6. doi:10.1136/jclinpath-2011200535

18. Román R, Verdú M, Calvo M, Vidal A, Sanjuan X, Jimeno M, et al. Microsatellite instability of the colorectal carcinoma can be predicted in the conventional pathologic examination. A prospective multicentric study and the statistical analysis of 615 cases consolidate our previously proposed logistic regression model. Virchows Arch (2010) 456(5):533-41. doi:10.1007/s00428-010-0896-6 
19. Halvarsson B, Anderson H, Domanska K, Lindmark G, Nilbert M. Clinicopathologic factors identify sporadic mismatch repair-defective colon cancers. Am J Clin Pathol (2008) 129(2):238-44. doi:10.1309/0PP5GDRTXUDVKAWJ

20. Compton C, Fenoglio-Preiser CM, Pettigrew N, Fielding LP. American joint committee on cancer prognostic factors consensus conference: colorectal working group. Cancer (2000) 88(7):1739-57. doi:10.1002/(SICI)10970142(20000401)88:7<1739::AID-CNCR30>3.0.CO;2-T

21. Jass JR, Atkin WS, Cuzick J, Bussey HJ, Morson BC, Northover JM, et al. The grading of rectal cancer: historical perspectives and a multivariate analysis of 447 cases. Histopathology (1986) 10(5):437-59. doi:10.1111/j.1365-2559.1986. tb02497.x

22. Jass JR, Love SB, Northover JM. A new prognostic classification of rectal cancer. Lancet (1987) 1(8545):1303-6. doi:10.1016/S0140-6736(87)90552-6

23. Jass JR, Ajioka Y, Allen JP, Chan YF, Cohen RJ, Nixon JM, et al. Assessment of invasive growth pattern and lymphocytic infiltration in colorectal cancer. Histopathology (1996) 28(6):543-8. doi:10.1046/j.1365-2559.1996.d01-467.x

24. Prall F. Tumour budding in colorectal carcinoma. Histopathology (2007) 50(1):151-62. doi:10.1111/j.1365-2559.2006.02551.x

25. Morikawa T, Kuchiba A, Qian ZR, Mino-Kenudson M, Hornick JL, Yamauchi M, et al. Prognostic significance and molecular associations of tumor growth pattern in colorectal cancer. Ann Surg Oncol (2012) 19(6):1944-53. doi:10.1245/ s10434-011-2174-5

26. Zlobec I, Terracciano LM, Lugli A. Local recurrence in mismatch repairproficient colon cancer predicted by an infiltrative tumor border and lack of CD8+ tumor-infiltrating lymphocytes. Clin Cancer Res (2008) 14(12):3792-7. doi:10.1158/1078-0432.CCR-08-0048

27. Horcic M, Koelzer VH, Karamitopoulou E, Terracciano L, Puppa G, Zlobec I, et al. Tumor budding score based on 10 high-power fields is a promising basis for a standardized prognostic scoring system in stage II colorectal cancer. Hum Pathol (2013) 44(5):697-705. doi:10.1016/j.humpath.2012.07.026

28. De Craene B, Berx G. Regulatory networks defining EMT during cancer initiation and progression. Nat Rev Cancer (2013) 13(2):97-110. doi:10.1038/nrc3447

29. Beaton C, Twine CP, Williams GL, Radcliffe AG. Systematic review and metaanalysis of histopathological factors influencing the risk of lymph node metastasis in early colorectal cancer. Colorectal Dis (2013) 15(7):788-97. doi:10.1111/ codi. 12129

30. Tanaka M, Hashiguchi Y, Ueno H, Hase K, Mochizuki H. Tumor budding at the invasive margin can predict patients at high risk of recurrence after curative surgery for stage II, T3 colon cancer. Dis Colon Rectum (2003) 46(8):1054-9. doi:10.1007/s10350-004-7280-z

31. Nakamura T, Mitomi H, Kanazawa H, Ohkura Y, Watanabe M. Tumor budding as an index to identify high-risk patients with stage II colon cancer. Dis Colon Rectum (2008) 51(5):568-72. doi:10.1007/s10350-008-9192-9

32. Wang LM, Kevans D, Mulcahy H, O'Sullivan J, Fennelly D, Hyland J, et al. Tumor budding is a strong and reproducible prognostic marker in T3N0 colorectal cancer. Am J Surg Pathol (2009) 33(1):134-41. doi:10.1097/PAS.0b013e318184cd55

33. Betge J, Kornprat P, Pollheimer MJ, Lindtner RA, Schlemmer A, Rehak P, et al. Tumor budding is an independent predictor of outcome in AJCC/UICC stage II colorectal cancer. Ann Surg Oncol (2012) 19(12):3706-12. doi:10.1245/s10434012-2426-z

34. Karamitopoulou E, Zlobec I, Kölzer V, Kondi-Pafiti A, Patsouris ES, Gennatas $\mathrm{K}$, et al. Proposal for a 10-high-power-fields scoring method for the assessment of tumor budding in colorectal cancer. Mod Pathol (2013) 26(2):295-301. doi:10.1038/modpathol.2012.155

35. Egashira Y, Yoshida T, Hirata I, Hamamoto N, Akutagawa H, Takeshita A, et al. Analysis of pathological risk factors for lymph node metastasis of submucosal invasive colon cancer. Mod Pathol (2004) 17(5):503-11. doi:10.1038/modpathol. 3800030

36. Keum MA, Lim SB, Kim SA, Yoon YS, Kim CW, Yu CS, et al. Clinicopathologic factors affecting recurrence after curative surgery for stage I colorectal cancer. J Korean Soc Coloproctol (2012) 28(1):49-55. doi:10.3393/jksc.2012.28.1.49

37. Akishima-Fukasawa Y, Ishikawa Y, Akasaka Y, Uzuki M, Inomata N, Yokoo T, et al. Histopathological predictors of regional lymph node metastasis at the invasive front in early colorectal cancer. Histopathology (2011) 59(3):470-81. doi:10.1111/j.1365-2559.2011.03964.X

38. Kajiwara Y, Ueno H, Hashiguchi Y, Mochizuki H, Hase K. Risk factors of nodal involvement in T2 colorectal cancer. Dis Colon Rectum (2010) 53(10):1393-9. doi:10.1007/DCR.0b013e3181ec5f66
39. Ueno H, Hase K, Hashiguchi Y, Ishiguro M, Kajiwara Y, Shimazaki H, et al. Growth pattern in the muscular layer reflects the biological behaviour of colorectal cancer. Colorectal Dis (2009) 11(9):951-9. doi:10.1111/j.1463-1318.2008. 01718.x

40. Zlobec I, Holler S, Tornillo L, Terracciano L, Lugli A. Combined histomorphologic and immunohistochemical phenotype to predict the presence of vascular invasion in colon cancer. Dis Colon Rectum (2009) 52(6):1114-21. doi:10.1007/DCR.0b013e31819eefd9

41. Cianchi F, Messerini L, Palomba A, Boddi V, Perigli G, Pucciani F, et al. Character of the invasive margin in colorectal cancer: does it improve prognostic information of Dukes staging? Dis Colon Rectum (1997) 40(10):1170-5; discussion 1175-6. doi:10.1007/BF02055162

42. Ueno H, Murphy J, Jass JR, Mochizuki H, Talbot IC. Tumour 'budding' as an index to estimate the potential of aggressiveness in rectal cancer. Histopathology (2002) 40(2):127-32. doi:10.1046/j.1365-2559.2002.01324.x

43. Cianchi F, Messerini L, Comin CE, Boddi V, Perna F, Perigli G, et al. Pathologic determinants of survival after resection of T3N0 (stage IIA) colorectal cancer: proposal for a new prognostic model. Dis Colon Rectum (2007) 50(9):1332-41. doi:10.1007/s10350-007-0222-9

44. Garcia-Solano J, Conesa-Zamora P, Trujillo-Santos J, Makinen MJ, PerezGuillermo M. Tumour budding and other prognostic pathological features at invasive margins in serrated colorectal adenocarcinoma: a comparative study with conventional carcinoma. Histopathology (2011) 59(6):1046-56. doi:10.1111/j.1365-2559.2011.04043.x

45. Huh JW, Lee JH, Kim HR. Prognostic significance of tumor-infiltrating lymphocytes for patients with colorectal cancer. Arch Surg (2012) 147(4):366-72. doi:10.1001/archsurg.2012.35

46. Greller LD, Tobin FL, Poste G. Tumor heterogeneity and progression: conceptual foundations for modeling. Invasion Metastasis (1996) 16(4-5):177-208.

47. Enderling H, Hlatky L, Hahnfeldt P. Tumor morphological evolution: directed migration and gain and loss of the self-metastatic phenotype. Biol Direct (2010) 5:23. doi:10.1186/1745-6150-5-23

48. Prehn RT. The inhibition of tumor growth by tumor mass. Cancer Res (1991) 51(1):2-4.

49. Rajaganeshan R, Prasad R, Guillou PJ, Chalmers CR, Scott N, Sarkar R, et al. The influence of invasive growth pattern and microvessel density on prognosis in colorectal cancer and colorectal liver metastases. Br J Cancer (2007) 96(7):1112-7. doi:10.1038/sj.bjc.6603677

50. Jass JR. HNPCC and sporadic MSI-H colorectal cancer: a review of the morphological similarities and differences. Fam Cancer (2004) 3(2):93-100. doi:10.1023/B:FAME.0000039849.86008.b7

51. Samowitz WS, Sweeney C, Herrick J, Albertsen H, Levin TR, Murtaugh MA, et al. Poor survival associated with the BRAF V600E mutation in microsatellite-stable colon cancers. Cancer Res (2005) 65(14):6063-9. doi:10.1158/0008-5472.CAN05-0404

52. Yokota T, Ura T, Shibata N, Takahari D, Shitara K, Nomura M, et al. BRAF mutation is a powerful prognostic factor in advanced and recurrent colorectal cancer. Br J Cancer (2011) 104(5):856-62. doi:10.1038/bjc.2011.19

53. Lochhead P, Kuchiba A, Imamura Y, Liao X, Yamauchi M, Nishihara R, et al Microsatellite instability and BRAF mutation testing in colorectal cancer prognostication. J Natl Cancer Inst (2013) 105(15):1151-6. doi:10.1093/jnci/djt173

54. Makrodouli E, Oikonomou E, Koc M, Andera L, Sasazuki T, Shirasawa S, et al BRAF and RAS oncogenes regulate Rho GTPase pathways to mediate migration and invasion properties in human colon cancer cells: a comparative study. $\mathrm{Mol}$ Cancer (2011) 10:118. doi:10.1186/1476-4598-10-118

55. Galon J, Costes A, Sanchez-Cabo F, Kirilovsky A, Mlecnik B, Lagorce-Pagès C, et al. Type, density, and location of immune cells within human colorectal tumors predict clinical outcome. Science (2006) 313(5795):1960-4. doi:10.1126/science.1129139

56. Mlecnik B, Tosolini M, Kirilovsky A, Berger A, Bindea G, Meatchi T, et al. Histopathologic-based prognostic factors of colorectal cancers are associated with the state of the local immune reaction. J Clin Oncol (2011) 29(6):610-8. doi:10.1200/JCO.2010.30.5425

57. Ogino S, Galon J, Fuchs CS, Dranoff G. Cancer immunology - analysis of host and tumor factors for personalized medicine. Nat Rev Clin Oncol (2011) 8(12):711-9. doi:10.1038/nrclinonc.2011.122

58. Pogue-Geile K, Yothers G, Taniyama Y, Tanaka N, Gavin P, Colangelo L, et al. Defective mismatch repair and benefit from bevacizumab for colon 
cancer: findings from NSABP C-08. J Natl Cancer Inst (2013) 105(13):989-92. doi:10.1093/jnci/djt140

59. Sargent DJ, Marsoni S, Monges G, Thibodeau SN, Labianca R, Hamilton SR, et al. Defective mismatch repair as a predictive marker for lack of efficacy of fluorouracil-based adjuvant therapy in colon cancer. J Clin Oncol (2010) 28(20):3219-26. doi:10.1200/JCO.2009.27.1825

60. Di Nicolantonio F, Martini M, Molinari F, Sartore-Bianchi A, Arena S, Saletti $\mathrm{P}$, et al. Wild-type BRAF is required for response to panitumumab or cetuximab in metastatic colorectal cancer. J Clin Oncol (2008) 26(35):5705-12. doi:10.1200/JCO.2008.18.0786

Conflict of Interest Statement: The authors declare that the research was conducted in the absence of any commercial or financial relationships that could be construed as a potential conflict of interest.
Received: 30 August 2013; accepted: 02 February 2014; published online: 18 February 2014.

Citation: Koelzer VH and Lugli A (2014) The tumor border configuration of colorectal cancer as a histomorphological prognostic indicator. Front. Oncol. 4:29. doi: 10.3389/fonc.2014.00029

This article was submitted to Gastrointestinal Cancers, a section of the journal Frontiers in Oncology.

Copyright () 2014 Koelzer and Lugli. This is an open-access article distributed under the terms of the Creative Commons Attribution License (CC BY). The use, distribution or reproduction in other forums is permitted, provided the original author(s) or licensor are credited and that the original publication in this journal is cited, in accordance with accepted academic practice. No use, distribution or reproduction is permitted which does not comply with these terms. 